\title{
Resonancia magnética cerebral con secuencia difusión - HASTE en la evaluación clínica del colesteatoma
}

\author{
Cerebral magnetic resonance with diffusion - HASTE sequence \\ for clinical evaluation of cholesteatoma
}

\author{
Eugenio Alzérreca $A^{1}$, Cristián Garrido $I^{2}$, Ricardo Zamorano $S^{3}$, Alvaro Salas $Z^{2}$ y Paul H Délano $R^{3,4}$.
}

\section{RESUMEN}

El diagnóstico del colesteatoma de oído es eminentemente clínico y su estudio complementario con imágenes se realiza con tomografía computarizada de cortes finos. En casos seleccionados de colesteatomas es necesario realizar un estudio con resonancia magnética (RM), utilizando habitualmente las secuencias T1 con gadolinio y T2. Se ha reportado alta especificidad y sensibilidad diagnóstica para colesteatoma con técnicas de difusión (DWI) en RM. Estas técnicas pueden ser montadas sobre secuencias no echo planares y rápidas, como la secuencia HASTE (Half Fourier Single Shot Turbo-spin Echo), que permiten obtener imágenes sin artefactos de susceptibilidad magnética en la interface entre el hueso y lóbulo temporal. En este trabajo se presenta una revisión de la literatura y cuatro casos clínicos evaluados en nuestro hospital en los que se utilizó la RM con secuencia DWI - HASTE. Se concluye que la RM con secuencia DWI - HASTE es una herramienta que permite tomar decisiones clínicas en un grupo seleccionado de pacientes con colesteatoma.

Palabras claves: Colesteatoma, resonancia magnética, HASTE, difusión.

\begin{abstract}
The diagnosis of ear cholesteatoma is done by clinical evaluation and its imaging study is performed mainly by computed tomography. In selected cases, it is necessary to consider a magnetic resonance imaging (MRI) in the evaluation of cholesteatoma, usually using gadolinium T1 and T2 sequences. It has been reported that MRI diffusion techniques (DWI) have high specificity and sensitivity results for the diagnostic of cholesteatoma. These techniques can be mounted on non-echo planar fast sequences, such as the HASTE sequence (Half Fourier Single-Shot Turbo Spin Echo), which allows the acquisition of images without any magnetic susceptibility artifacts at the interface between bone and the temporal lobe. This paper presents a literature review on the topic, and four clinical cases evaluated in our hospital, where MRI was used with DWI - HASTE sequence. We conclude that MRI with DWI - HASTE sequence is a useful tool for making clinical decisions in the assessment of a selected group of patients with cholesteatoma.
\end{abstract}

Key words: Cholesteatoma, Magnetic Resonance Imaging, Diffusion Weighted Imaging, HASTE.

\footnotetext{
${ }^{1}$ Magister en Derecho de la Salud. Universidad de los Andes.

${ }^{2}$ Servicio de Radiología. Hospital Clínico Universidad de Chile.

${ }^{3}$ Servicio de Otorrinolaringología. Hospital Clínico Universidad de Chile.

${ }^{4}$ Programa de Fisiología y Biofísica, ICBM, Facultad de Medicina, Universidad de Chile.
} 


\section{INTRODUCCIÓN}

El diagnóstico del colesteatoma de oído se realiza con una evaluación clínica del paciente que incluye anamnesis, otomicroscopía y audiometría. La tomografía computada (TC) de oídos con corte fino $(0,5 \mathrm{~mm})$ es el examen de elección para el estudio con imágenes del paciente con diagnóstico de colesteatoma. La TC puede mostrar signos sugerentes de colesteatoma como opacidad de la caja timpánica, erosión de la pared lateral del epitímpano o de los huesecillos del oído medio y signos de complicación como dehiscencia del tegmen timpani, del canal semicircular lateral 0 compromiso del nervio facial ${ }^{1}$.

En casos seleccionados, la resonancia magnética (RM) tiene un valor adicional en describir posibles complicaciones del colesteatoma, como la invasión de laberinto membranoso o la extensión intracraneana con afectación de tejido cerebral ${ }^{1,2}$. Habitualmente se solicita una RM de cerebro con gadolinio, utilizando la secuencia $\mathrm{T} 1$ con gadolinio (T1-Gd) y T2. En la T1-Gd un colesteatoma se observa como una señal isointensa que no capta gadolinio y se encuentra rodeado por un halo hiperintenso (capta Gd), que corresponde al tejido inflamatorio que envuelve al colesteatoma. En T2 se observa al colesteatoma como una señal de alta intensidad, similar al líquido cerebro espinal.

Se ha reportado que las técnicas de difusión en RM (DWI) serían especialmente sensibles en la detección de colesteatomas de oído ${ }^{2-4}$. Las técnicas de DWI, ampliamente utilizadas en el estudio de pacientes con accidentes cerebro-vasculares, se pueden obtener a través de secuencias echoplanares y no echo planares. Las técnicas de DWI con imágenes echo planares presentan con alta frecuencia artefactos de distorsión geométrica y susceptibilidad magnética que se acentúan al explorar la base de cráne $0^{4}$.

En los últimos años se ha introducido una secuencia de DWI no echo planar para el estudio del colesteatoma de oído, denominada "HalfFourier acquisition single-shot turbo-spin-echo", que se abrevia como secuencia HASTE. Esta técnica de difusión permite diferenciar tejido inflamatorio o granulatorio del tejido colesteatomatoso, debido a su alta potenciación en DWI con secuencia T2, lo que permite visualizar el colesteatoma como una imagen de alta intensidad debido a su contenido de queratina ${ }^{5}$. Se han reportado valores predictivos positivos (VPP) y valores predictivos negativos (VPN) con RM más secuencia DWI HASTE entre $75 \%$ a $100 \%$, tanto en el estudio primario de un colesteatoma como en pacientes operados para buscar enfermedad residual ${ }^{6}$. A continuación se presenta la experiencia de cuatro pacientes evaluados por colesteatoma en nuestro hospital con la utilización de RM con método de DWI sobre una secuencia HASTE.

\section{CASOS CLÍNICOS}

\section{Caso 1}

Paciente de 15 años de edad, de sexo masculino, con antecedentes de traumatismo encéfalo craneano abierto a los 5 años, con fractura de hueso temporal del oído izquierdo más pérdida de masa encefálica y reparación de escama del hueso temporal con placa de acrílico. Consulta en policlínico de otorrinolaringología (ORL) por cuadro de hipoacusia progresiva de oído izquierdo. La audiometría muestra audición normal del oído derecho e hipoacusia conductiva de oído izquierdo (PTP vía aérea/ósea: $65 / 20 \mathrm{~dB}$ ). Al examen físico destaca una otoscopía que evidencia la presencia de una masa de características epiteliales en conducto auditivo externo de oído izquierdo, que impide visualizar el tímpano, por lo que se decide tomar biopsia de tejido que es informada como laminillas corneas compatibles con colesteatoma. Se solicita estudio de imágenes con TC que informa ocupación mastoidea izquierda y dehiscencia del tegmen timpanicon amplia comunicación entre oído medio y fosa cerebral media. Se solicita RM con secuencia T1-Gd, T2 y DWI - HASTE (Figura 1). La RM muestra colesteatoma rodeando a meningocele que desciende desde fosa media a cavidad timpánica. Se diagnostica un colesteatoma postraumático y se programa para cirugía radical de oído izquierdo en conjunto con neurocirugía, donde se confirma la presencia de colesteatoma y meningocele. En el posoperatorio el paciente evolucionó con otorraquia por conducto auditivo izquierdo por lo que se programó para reparación de 
fístula posquirúrgica con reparación mediante músculo y fascia crural.

\section{Caso 2}

Paciente de 57 años de edad, de sexo femenino con antecedentes de hipertensión arterial en tratamiento. Consulta a policlínico de ORL con historia de cofosis de oído izquierdo y parálisis facial izquierda de dos meses de evolución. Además relata crisis de vértigo y desequilibrio postural meses atrás. Al examen físico destaca una parálisis facial izquierda completa grado VI (índice House-Brackmann), más una otoscopía que evidencia un tumor que desplaza tímpano y pared inferior del conducto auditivo externo, asociado a otorrea purulenta de oído izquierdo. La audiometría muestra una audición normal del oído derecho (PTP vía aérea/ósea: 13/12 dB) y cofosis del oído izquierdo. Además se realiza estudio con TC de oídos (Figura $2 \mathrm{~A})$ y RM cerebral preoperatoria, los cuales fueron informados como sugerentes de proceso expansivo con compromiso de ángulo pontocerebeloso izquierdo, diagnosticándose un colesteatoma, con sospecha de carcinoma de oído por lo que se programa para cirugía radical de oído izquierdo. En la cirugía se comprueba el diagnóstico de colesteatoma con extensión al ángulo pontocerebeloso que es extirpado en forma parcial. No se encuentran elementos de malig-

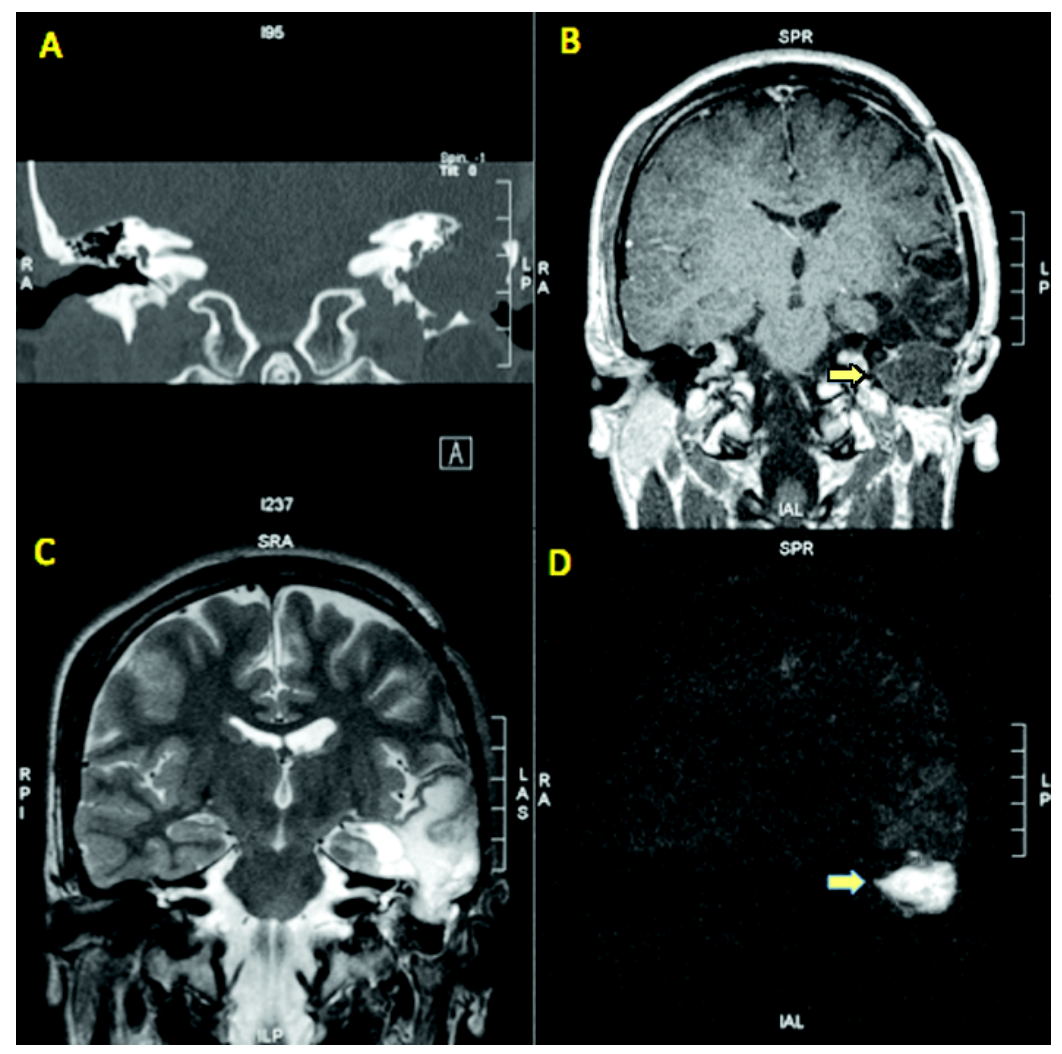

Figura 1. Estudio preoperatorio con tomografía computarizada y resonancia magnética del caso clínico $n^{0} 1$. A) Tomografía computarizada de oído. Se muestra una imagen de reconstrucción coronal realizada antes de la cirugía de extirpación del colesteatoma. Se observa ocupación de mastoides izquierda con tejido de densidad de partes blandas que está ampliamente comunicado con la fosa media cerebral. B) Resonancia magnética obtenida en secuencia T1 con gadolinio. Adquisición coronal. La flecha muestra imagen de lesión isointensa en el peñasco izquierdo con dehiscencia hacia fosa media. Nótese el refuerzo periférico que se produce en la lesión por la captación de gadolinio por el tejido inflamatorio. C) RM en secuencia T2. Adquisición coronal. La imagen evidencia ocupación con señal intermedia e hiperintensa, coincidente con dehiscencia desde fosa media con descenso parcial de tejido cerebral temporal y presencia de colesteatoma indistinguible del tejido cerebral D) RM con secuencia DWI-HASTE. Adquisición coronal. La flecha muestra una lesión hiperintensa en peñasco compatible con colesteatoma. Se observa imagen sugerente de meningocele ótico que se confirmó en la cirugía. 

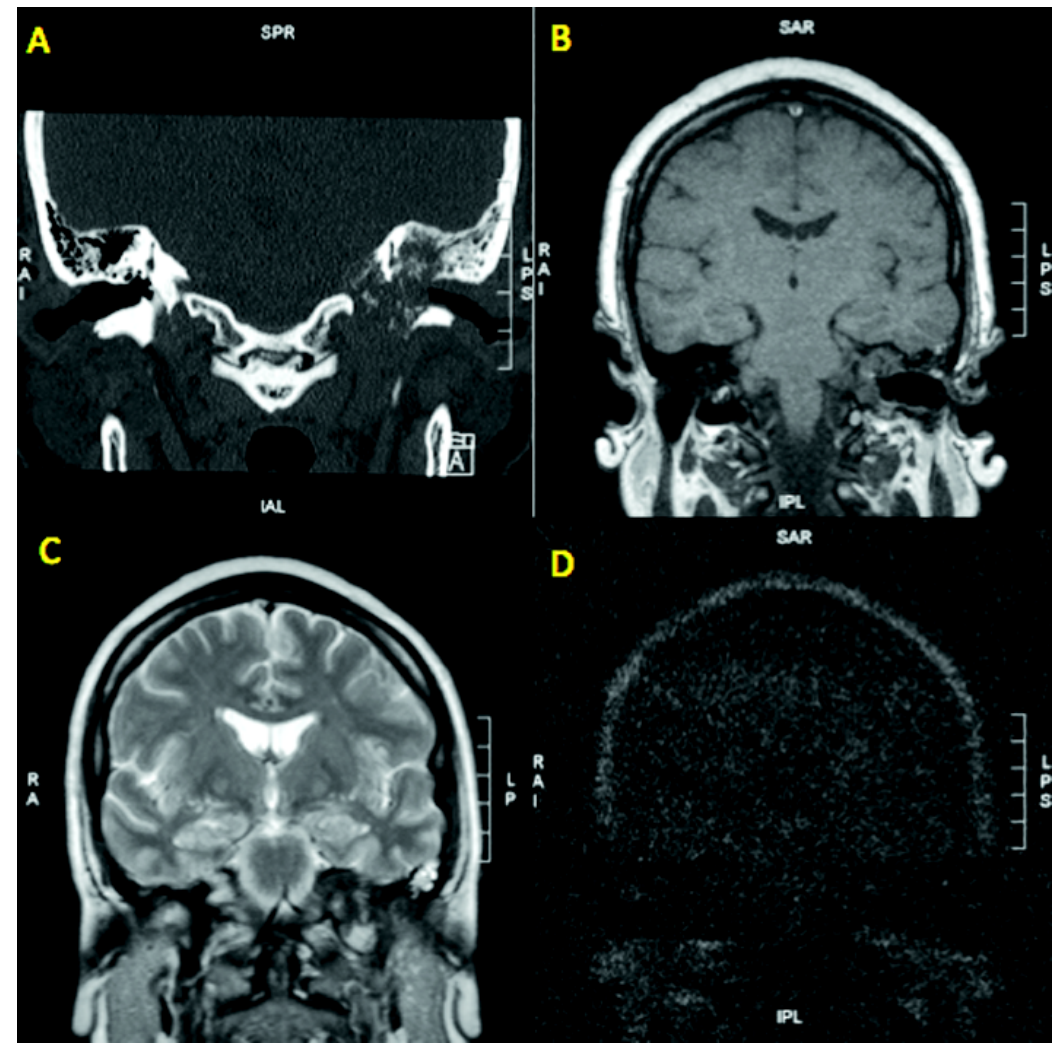

D

SAR

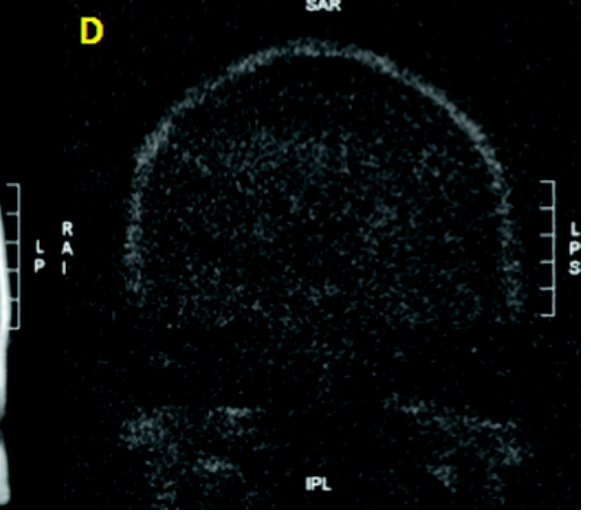

Figura 2. Estudio pre y posoperatorio con tomografía computarizada y resonancia magnética del caso clínico $n^{0} 2$. A) TC de oído preoperatoria en reconstrucción coronal. Se observa lesión isointensa con restos de erosión ósea en oído izquierdo, que se extiende a la fosa cerebral posterior. B) RM en secuencia T1 (posoperatorio). Adquisición coronal. C) RM en secuencia T2. Adquisición coronal. Tanto en T1 como en T2 se evidencian cambios posquirúrgicos en el oído izquierdo. D) RM con secuencia DWI-HASTE (posoperatorio). Adquisición coronal. Se aprecia la ausencia de señal hiperintensa característica del colesteatoma.

nidad en el estudio histopatológico del colesteatoma. Posteriormente, la paciente es controlada en forma mensual para limpieza de cavidad radical por presentar colesteatoma residual. Dos años después de la cirugía la paciente refiere cefalea persistente de 2 meses de evolución, por lo que se solicita estudio de RM con secuencia DWI - HASTE para evaluar la posibilidad de crecimiento intracraneano del remanente de colesteatoma (Figura 2D). Este estudio no muestra signos de crecimiento intracraneal del colesteatoma, por lo que se decide mantener una conducta expectante y mantener las limpiezas mensuales de la cavidad radical.

\section{Caso 3}

Paciente de 46 años de edad, sexo masculino, con antecedentes de una timpanoplastía con injerto de fascia por una otitis media crónica (OMC) de oído izquierdo. Consulta a policlínico de ORL por cuadro de 2 meses de evolución caracterizado por otorrea y tinitus del oído izquierdo. Al examen micro-otoscópico del oído izquierdo se observa perforación antero inferior del injerto con presencia de piel en caja timpánica. Se diagnostica OMC colesteatomatosa y se realiza cirugía radical de oído izquierdo. En controles posoperatorios evoluciona con otorrea y tinitus de oído izquierdo. Una otomicroscopía demuestra una cavidad radical con injerto parcialmente lateralizado y con escasa otorrea que remite al tratamiento antibiótico. Se solicita TC de oídos posoperatorio que demuestra velamiento parcial a nivel de caja timpánica (Figura 3A), por lo que se decide presentar el caso en comité de oídos, que sugiere controlar clínicamente y con TC de oídos cada 6 meses. Sin embargo, ante la persistencia del cuadro, se sospecha colesteatoma residual y se 


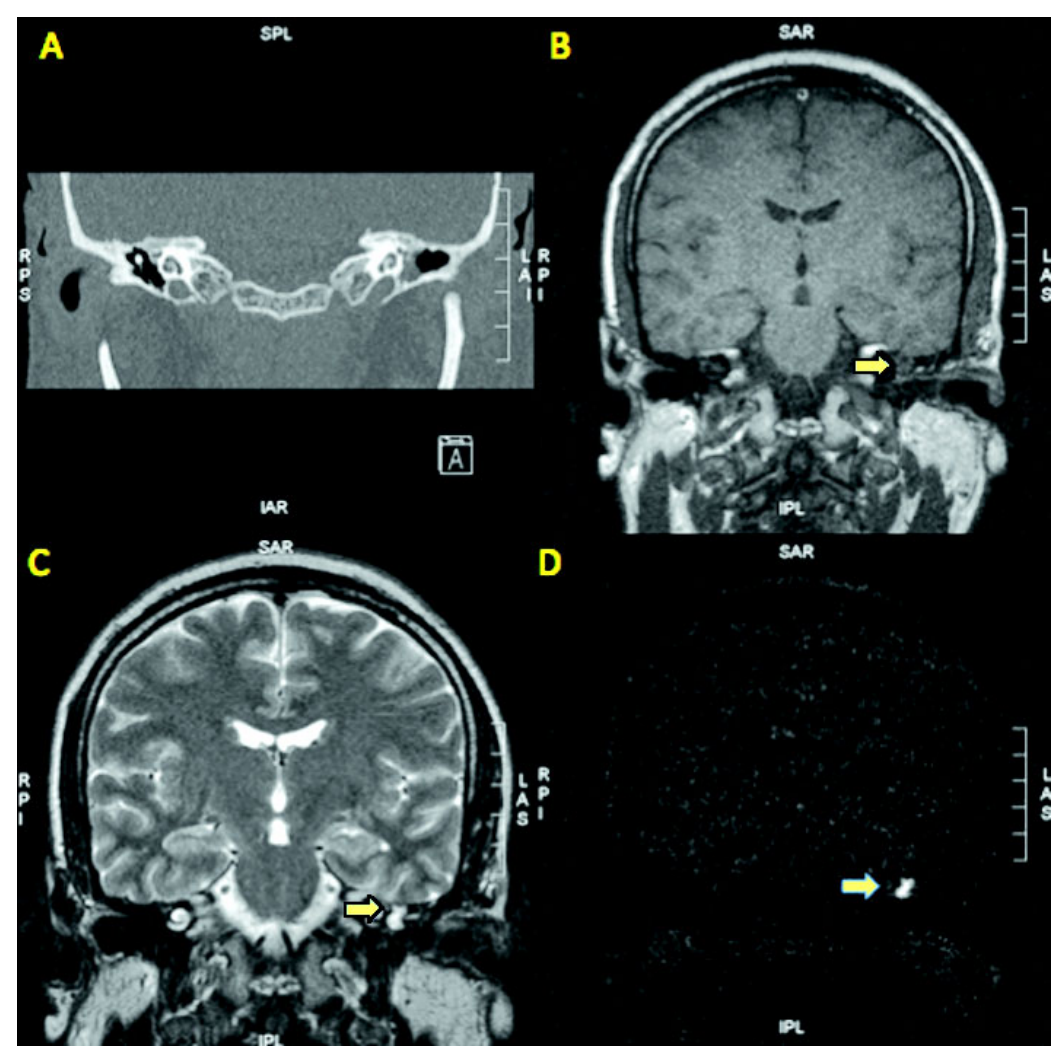

Figura 3. Tomografía computarizada y resonancia magnética obtenidas después de cirugía radical modificada de oído izquierdo en el caso clínico $n^{0} 3$. A) TC oído en reconstrucción coronal. Opacidad del protímpano a nivel de la tuba auditiva izquierda B) $\mathrm{RM}$ en secuencia T1. Adquisición coronal. La flecha muestra imagen hipointensa de oído izquierdo indistinguible de tejido peri inflamatorio y posquirúrgico. C) RM en secuencia T2. Adquisición coronal. La flecha muestra imagen hiperintensa que podría corresponder a un colesteatoma recidivado. D) RNM con secuencia DWI-HASTE. Adquisición coronal. La flecha muestra una lesión hiperintensa compatible con colesteatoma que se confirmó durante la cirugía de revisión.

solicita estudio de imágenes con RM con secuencia DWI - HASTE, que demuestra tejido colesteatomatoso residual en protímpano del oído izquierdo (Figura 3D). Se realiza cirugía de revisión del oído izquierdo, que confirma la presencia del colesteatoma según los hallazgos de la RM con DWI - HASTE.

\section{Caso 4}

Paciente de 62 años de edad, de sexo femenino, sin antecedentes mórbidos. Consulta por cuadro de 5 años de evolución caracterizado por hipoacusia y otorrea bilateral intermitente. Al examen físico, la otoscopía de oído derecho evidencia un fondo de saco de tejido inflamatorio que no permite visualizar tímpano y perforación timpánica del oído izquierdo. Se solicita estudio con audiometría que informa una hipoacusia mixta bilateral y una TC de oído que informa ocupación de caja timpánica bilateral por material hipodenso, con erosión de escutum en oído derecho y de la cadena osicular a izquierda (Figura 4A). Se realiza el diagnóstico de una OMC colesteatomatosa del oído derecho y se cita a comité de oído, donde se decide realizar una RM con secuencia DWI - HASTE, la cual es informada como libre de evidencia de tejido colesteatomatoso (Figura 4D).

\section{DISCUSIÓN}

Las diferentes técnicas de RM han tenido un desarrollo sustancial en la última década. La técnica de difusión para RM se basa en el estudio del movimiento de las moléculas de agua en los diferentes tejidos, permitiendo la diferencia de 


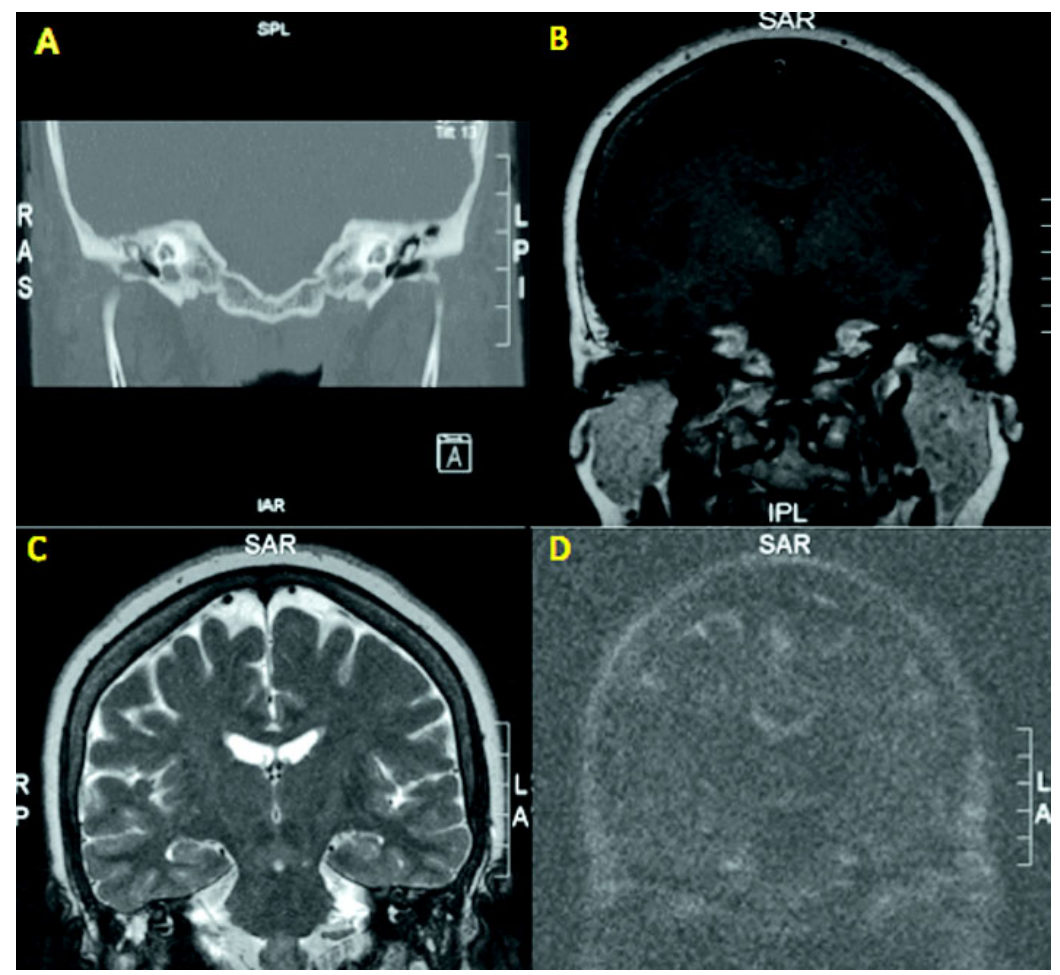

Figura 4. Tomografía computarizada y resonancia del caso clínico n4. A) TC de oído. Reconstrucción coronal. Se evidencia ocupación de caja timpánica derecha por tejido hipodenso, con erosión del scutum en oído derecho B) RM en secuencia T1. Adquisición coronal C) RM en secuencia T2. Adquisición coronal D) RM con secuencia DWI-HASTE. Adquisición coronal. Se observa ausencia de señal hiperintensa característica de colesteatoma.

éstos según su contenido acuoso. Reportes recientes sugieren que el uso de técnicas de difusión montadas sobre secuencias echo planares, han permitido la obtención de imágenes multiplanares rápidas en regiones como la fosa posterior, lóbulo frontal y lóbulo temporal, sin embargo, mantienen una alta tasa de artefactos de distorsión geométrica y susceptibilidad magnética, sobre todo en regiones de interface entre cerebro y aire como entre el hueso y lóbulo temporal ${ }^{5}$.

La secuencia DWI - HASTE, utilizada en los casos de pacientes descritos, corresponde a una técnica no echo planar, que permite obtener imágenes rápidas, con buena resolución y con menos distorsión y artefactos, sobre todo presentes en el estudio de base de cráneo, al compararlos con las secuencias DWI echo planares.

En este reporte de casos, se presentaron 4 pacientes con historia de colesteatoma, cuyos resultados de imágenes obtenidas en RM con secuencia DWI -
HASTE permitieron tomar diferentes conductas. En dos casos (casos 1 y 3), se decidió llevar a cabo cirugías que confirmaron la presencia de colesteatomas, mientras que en los casos 2 y 4 se decide observar, no operar y controlar clínicamente a los pacientes (Tabla 1).

Estudios realizados por diversos autores muestran que la RM con secuencia DWI - HASTE tiene un alto valor predictivo positivo y negativo, tanto para el estudio preoperatorio de cirugía primaria como para el estudio de revisión de colesteatoma. En la publicación de Dhepnorrarat y cols ${ }^{4}$ (2009), se evaluó la presencia de colesteatoma con secuencia DWI HASTE mensual entre 3 a 6 meses antes de la segunda cirugía de revisión y se describieron las características en RM del edema, fluido seroso y tejido granulatorio posquirúrgico. Se demostró un VPP de $100 \%$ y un VPN de $100 \%$ en la detección de colesteatoma residual luego de cirugía primaria. La sensibilidad y especificidad del estudio fue de $100 \%$ para detección de colesteatoma. 
Tabla 1: Características clínicas de los pacientes

\begin{tabular}{|c|c|c|c|c|c|}
\hline Paciente & Sexo & Edad & Tipo colesteatoma & Hallazgos DWI-HASTE & Conducta según DWI-HASTE \\
\hline 1 & M & 15 años & $\begin{array}{l}\text { Colesteatoma primario } \\
\text { postraumático }\end{array}$ & $\begin{array}{l}\text { Confirma diagnóstico } \\
\text { de colesteatoma }\end{array}$ & $\begin{array}{l}\text { Cirugía que confirma } \\
\text { colesteatoma }\end{array}$ \\
\hline 2 & $\mathrm{~F}$ & 57 años & $\begin{array}{l}\text { Colesteatoma remanente } \\
\text { posquirúrgico }\end{array}$ & $\begin{array}{l}\text { Ausencia de tejido } \\
\text { remanente colesteatomatoso }\end{array}$ & Observación clínica \\
\hline 3 & M & 46 años & $\begin{array}{l}\text { Colesteatoma remanente } \\
\text { posquirúrgico }\end{array}$ & $\begin{array}{l}\text { Confirma presencia de } \\
\text { remanente colesteatomatoso }\end{array}$ & $\begin{array}{l}\text { Cirugía que confirma } \\
\text { remanente de colesteatoma }\end{array}$ \\
\hline 4 & $\mathrm{~F}$ & 62 años & Sospecha de colesteatoma & $\begin{array}{l}\text { Ausencia de tejido } \\
\text { colesteatomatoso }\end{array}$ & Observación clínica \\
\hline
\end{tabular}

En el estudio de Pizzini y cols $^{7}$ (2010), se evaluaron 30 pacientes con sospecha de colesteatoma adquirido primario o recurrente, realizando RM con secuencia DWI - HASTE con un equipo resonador de 3.0 Tesla. Se obtuvo un VPP y un VPN de $100 \%$ tanto para los hallazgos quirúrgicos en cirugía de colesteatoma primario como de colesteatoma recurrente. El uso de equipos resonadores con mayor potencia en Tesla, permite generar mayores campos magnéticos para el ordenamiento de los protones en las moléculas de agua y así obtener imágenes de mayor resolución, sin embargo, en el estudio de colesteatomas con secuencia DWI - HASTE, la nitidez anatómica de las imágenes adquiridas no es lo más importante al tratarse de una técnica de difusión.

Existe también una buena correlación interobservador entre radiólogos que interpretan las imágenes del estudio con RM más secuencia DWI - HASTE. En el estudio de De Foer y cols $^{3}$ (2010), se revisaron en forma retrospectiva 57 pacientes con cirugía primaria de colesteatoma y 63 pacientes con cirugía de revisión por colesteatoma, comparando sensibilidad, especificidad, VPP y VPN de RM con secuencia T1-Gadolinio versus DWI - HASTE. Se obtuvo un coeficiente kappa de correlación interobservador para secuencia T1-Gd de 0,363; un coeficiente kappa de 0,788 para secuencia DWI - HASTE y un coeficiente de 0,781 para ambas técnicas combinadas. De acuerdo a los resultados de este estudio, no sería necesario realizar un estudio con secuencia $\mathrm{T} 1$ con uso de gadolinio como contraste, puesto que el coeficiente obtenido con técnica DWI - HASTE es similar a la de ambas técnicas combinadas.
Estas características de la secuencia DWI HASTE, las cuales permiten obtener imágenes de adecuada resolución y diferenciar con mayor exactitud entre tejido colesteatomatoso propiamente tal y tejido granulatorio o inflamatorio, la ha convertido en una herramienta de gran ayuda en la detección del colesteatoma residual, determinando muchas veces que no sea necesaria una nueva cirugía de revisión.

Una limitación existente en el estudio imagenológico de colesteatoma con RM más secuencia DWI - HASTE, son aquellos casos donde existen bolsillos de retracción o colesteatomas con autoevacuación y escaso contenido de queratina, resultando en colesteatomas secos, los que no producen señales de hiperintensidad en el estudio, impidiendo su detección $n^{4,7,8}$. De Foer y cols ${ }^{2}$ (2007), evaluaron prospectivamente a 21 pacientes con alta sospecha clínica de colesteatoma, mediante RM con secuencia DWI - HASTE, comparando los hallazgos radiológicos con los quirúrgicos, obteniendo una detección imagenológica de colesteatomas con un tamaño igual o mayor a 2 $\mathrm{mm}$. Ellos reportan dos casos de falsos negativos, uno de los cuales correspondió a un bolsillo de retracción con piel.

También se ha reportado que la RM con secuencia DWI - HASTE tendría un posible beneficio para el estudio de colesteatomas en niños, por el menor tiempo de duración del examen y la posibilidad de evitar el uso de medio de contraste. En el estudio realizado por Rajan y $\operatorname{cols}^{9}(2010)$, con niños entre 2 a 16 años, el tiempo requerido para realizar la secuencia DWI - HASTE fue de 100 segundos en promedio a diferencia de los 20 minutos requeridos 
en las secuencias estándar. Debido a esto ninguno de los pacientes recibió anestesia o sedación en el procedimiento imagenológico. En este estudio se obtuvo un VPP de $100 \%$ y un VPN de $100 \%$ para detección de colesteatoma, con una sensibilidad y especificidad de $100 \%$.

\section{CONCLUSIONES}

El diagnóstico del colesteatoma de oído sigue siendo eminentemente clínico y su estudio complementario puede ser apoyado por imágenes con TC de oído de cortes finos. Si bien la RM no es un examen que se deba solicitar a todos los pacientes con sospecha de colesteatoma primario, los altos VPP y VPN hacen que la utilización de la secuencia DWI - HASTE en protocolos de RM, deba ser considerada en aquellos pacientes con colesteatomas recurrentes.

La literatura actual, sumada a la experiencia en nuestro hospital nos permite sugerir la solicitud del estudio de RM cerebral con secuencia DWI HASTE en el Servicio de Imagenología del Hospital Clínico de la Universidad de Chile. Nos permitimos sugerir las siguientes indicaciones del estudio de RM con secuencia DWI - HASTE:

(i) posibles colesteatomas congénitos;

(ii) evaluación de colesteatomas en niños;

(iii) el estudio previo a una cirugía de revisión;

(iv) la evaluación de colesteatomas que comprometen tejido cerebral o base de cráneo.

\section{BIBLIOGRAFÍA}

1. De Foer B, Vercruysse JP, Pilet B. Single-shot, turbo spin-echo, diffusion-weighted imaging versus spin-echo-planar, diffusion-weighted imaging in the detection of acquired middle ear cholesteatoma. AJNR Am J Neuroradiol 2006; 27(7): 1480-2.
2. De Foer $B$, Vercruysse JP, Bernaerts $A$. The value of single-shot turbo spin-echo diffusionweighted MR imaging in the detection of middle ear cholesteatoma. Neuroradiology 2007; 49(10): 841-8.

3. De Foer B, Vercruysse JP, Bernaerts A. Middle Ear Cholesteatoma: Non-Echo-planar Diffusion-weighted MR Imaging versus Delayed Gadolinium-enhanced T1-weighted MR Imaging - Value in Detection. Radiology 2010; 255: 866-72.

4. Dhepnorrarat RC, Wood B, Rajan GP. Postoperative Non-Echo-Planar Diffusion-Weighted Magnetic Resonance Imaging Changes After Cholesteatoma Surgery: Implications for Cholesteatoma Screening. Otol Neurotol 2009; 30(1): 54-8.

5. Dubrulle F, Souillard R, Chechin D. Diffusionweighted MR Imaging Sequence in the Detection of Postoperative Recurrent Cholesteatoma. Radiology 2006; 238: 604-10.

6. Huins $C$, Singh A, Kumar R. Detecting cholesteatoma with non-echo planar (HASTE) diffusion-weighted magnetic resonance Imaging. Otolaryngol Head Neck Surg 2010; 143(1): 141-6.

7. Pizzini FB, Barbieri F, Beltramello A. HaSte diffusion-weighted 3-Tesla magnetic resonance imaging in the diagnosis of primary and relapsing cholesteatoma. Otol Neurotol 2010; 31(4): 596-602.

8. Lehmann P, Saliou G, Brochart C. 3T MR imaging of postoperative recurrent middle ear cholesteatomas: value of periodically rotated overlapping parallel lines with enhanced reconstruction diffusion-weighted MR imaging. AJNR Am J Neuroradiol 2009; 30(2): 423-7.

9. Rajan GP, Ambett R, Wun L. Preliminary outcomes of cholesteatoma screening in children using non-echo-planar diffusionweighted magnetic resonance imaging. Int $J$ Pediatr Otorhinolaryngol 2010; 74(3): 297-301.

\footnotetext{
Dirección: Dr. Paul H. Délano R.

Servicio Otorrinolaringología, Hospital Clínico Universidad de Chile

Santos Dumont, 999 Santiago, Chile

E mail: pdelano@med.uchile.cl
} 